САХАРНЫЙ ДИАБЕТ 2 ТИПА. КОМБИНИРОВАННАЯ ТЕРАПИЯ НА СТАРТЕ ЗАБОЛЕВАНИЯ

(c) Francesco Indovina, Pierpaolo Falcetta, Stefano Del Prato*

Department of Clinical and Experimental Medicine, Section of Metabolic Diseases and Diabetes, University of Pisa, Пиза, Италия

Современное лечение сахарного диабета 2 типа (СД2) требует нового подхода в усилении терапии с момента постановки диагноза. Это подтверждается данными, показывающими как даже небольшая задержка в достижении хорошего гликемического контроля может способствовать повышению риска развития диабетических осложнений. Признание сложности патогенеза СД2 приводит к пониманию важности одновременного воздействия на несколько механизмов, способствующих гипергликемии. С начала века появилось несколько новых групп сахароснижающих препаратов. По сравнению со старыми, эти лекарства имеют более направленный механизм действия, поскольку они действуют на уровне специфических патофизиологических нарушений, учитывающих развитие и прогрессирование гипергликемии. В связи с этим препараты могут быть применены в комбинации для использования их дополнительных механизмов действия. В данной работе мы обсудим преимущества, недостатки и еще нерешенные вопросы, связанные с использованием ранней комбинированной терапии при СД2.

КЛЮЧЕВЫЕ СЛОВА: сахарный диабет 2 типа; ранняя комбинированная сахароснижающая терапия; терапия; патофизиология

\title{
TYPE 2 DIABETES MELLITUS. FROM THE START - COMBINATION THERAPY
}

(c) Francesco Indovina, Pierpaolo Falcetta, Stefano Del Prato*

Department of Clinical and Experimental Medicine, Section of Metabolic Diseases and Diabetes, University of Pisa, Pisa, Italy

Modern treatment of T2DM requires a shift in paradigm with appropriate intensification of therapy from the very first time of diabetes diagnosis. This is supported by data showing how even a moderate delay in achieving good glycemic control can translate into a later increased risk of developing diabetic complications. The recognition of the complexity of the pathogenesis of T2DM leads to the appreciation of the importance of attacking the disease from different angles, i.e. simultaneous tackling of multiple mechanisms contributing to hyperglycemia. From the turn of century a growing number of new anti-hyperglycemic agents have been made available. As compared to the older ones, these new medicines have a more targeted mechanism of action as they act at the level of the specific pathophysiologic disturbances accounting the development and progression of hyperglycemia. Because of that drugs can be use in combination taking advantage of their complementary mechanisms of action and synergistic. If introduced earlier in the natural history of the disease combination therapy may contribute avoiding undesirable exposure to even mild chronic hyperglycemia and provide early benefits. With respect to that in this review we will discuss advantages, disadvantages and still unanswered questions related to the use of early combination therapy in type 2 diabetes.

KEY WORDS: type 2 diabetes; therapy; early combination; pathophysiology

\section{ВВЕДЕНИЕ}

Фармакологический арсенал для лечения сахарного диабета 2 типа (СД2) значительно расширился за последние 20-30 лет. После десятилетий лекарственной «стагнации», во время которой сахароснижающие возможности были основаны на бигуанидах, производных сульфонилмочевины и более старых препаратах инсулина, к началу века появилось множество новых классов сахароснижающих препаратов, доступных для применения [1] (рис. 1). Однако эта революция, похоже, не соотносится с увеличением количества пациентов с СД2, достигающих и, что более важно, поддерживающих хороший контроль гликемии. Недавний анализ данных Национального опроса для оценки здоровья и питания (NHANES) по 2677 взрослым с 2007 по 2014 гг. показал, что доля пациентов с диабетом и гликированным ге- моглобином $\left(\mathrm{HbA}_{1 c}\right)<7,0 \%$ немного снизилась - с 52,2\% до 50,9\% между двумя последними оценками в базе данных. Даже после оценки достижения индивидуальных целей лечения (в зависимости от возраста и осложнений), за тот же период времени снижение составило от $69,8 \%$ до 63,8\%. Хуже того, процент пациентов с $\mathrm{HbA}_{1 с}$ $>9,0 \%$ вырос с 12,6\% до 15,5\% [2]. Причина неполного успеха, несмотря на разработку большого количества новых лекарств, имеет множество объяснений.

\section{КЛИНИЧЕСКАЯ «ИНЕРТНОСТЬ" В ЛЕЧЕНИИ ПАЦИЕНТОВ С САХАРНЫМ ДИАБЕТОМ 2 ТИПА}

Среднее время консультации пациента с диабетом не занимает более 10 мин. В настоящее время слишком мало времени на консультациях больных посвящается должному взаимодействию с пациентами и быстрой оценке 


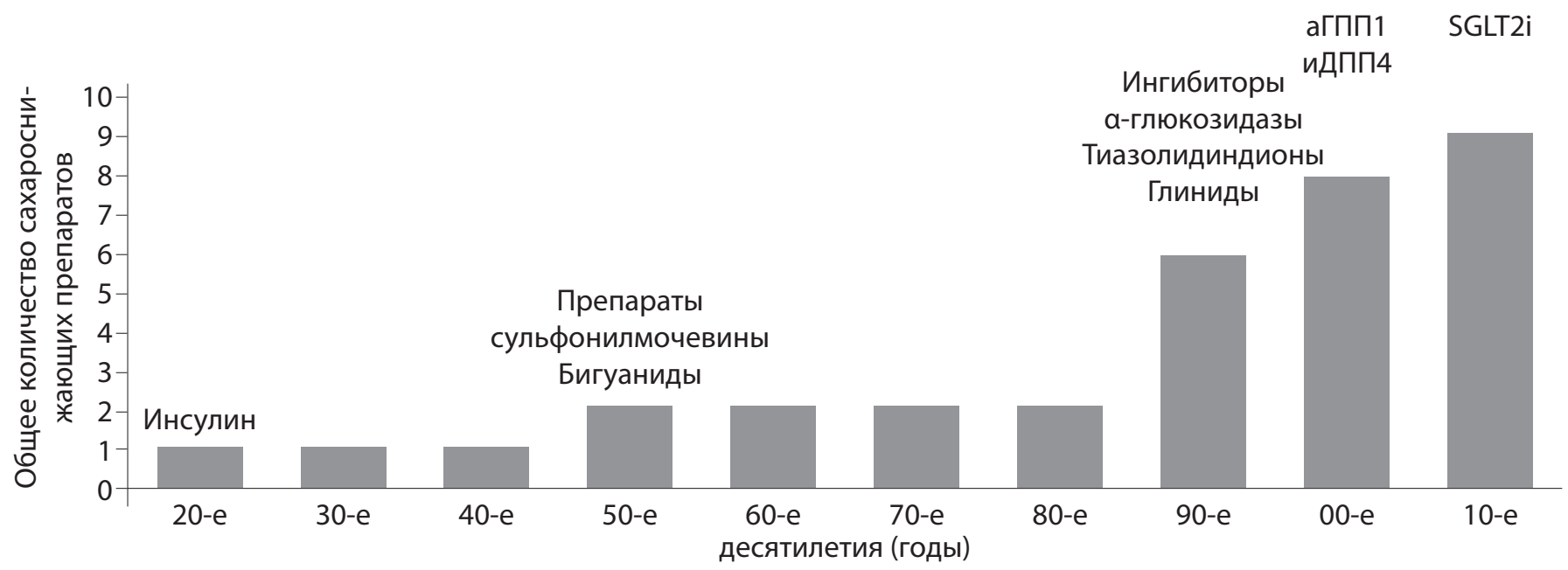

Рис. 1. Развитие фармакологического арсенала с течением времени.

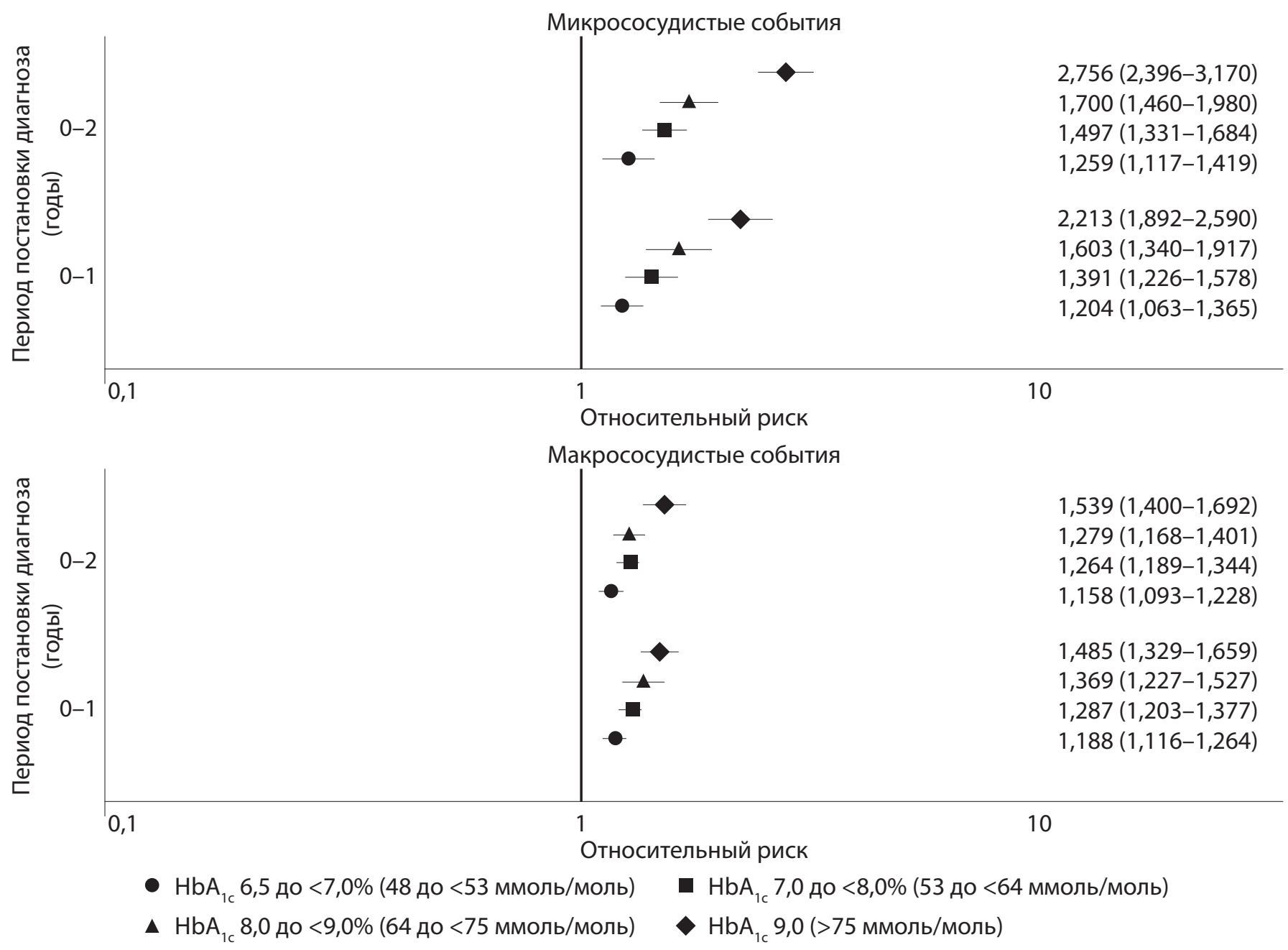

Рис. 2. Отношения рисков в сравнении частоты микрососудистых (верхняя часть) и макрососудистых (нижняя часть) событий при различном уровне $\mathrm{HbA}_{1 с}$ в первый год постановки диагноза и в первые два года после установления диагноза.

Уровни сравниваются с показателем <6,5\% (<48 ммоль/моль) в том же периоде наблюдения. Отношения рисков, скорректированные по году постановки диагноза, возрасту на момент постановки диагноза, полу, расе/национальности, индексу массы тела, систолическому и диастолическому артериальному давлению, общему холестерину, холестерину липопротеидов высокой плотности, курению, НbА наблюдения и коморбидности. (Адаптировано [4]).

необходимости изменения или интенсификации лечения. Эти ограничения являются основной причиной клинического застоя. В ретроспективном когортном исследовании среди 81573 пациентов с СД2 Khunti и соавт. [3] продемонстрировали, что у пациентов с $\mathrm{HbA}_{1 c}<7,0,<7,5$ или $<8,0 \%$ ( $<53,<58$ или $<64$ ммоль/моль) среднее время до интенсификации лечения составляло 2,9, 1,9 или 1,6 года соответственно среди пациентов, получающих один пероральный сахароснижающий препарат (ПССП), и >7,2, >7,2 и >6,9 года соответственно среди получавших два ПССП. Среднее время до интенсификации у пациентов, получавших инсулинотерапию, было >7,1, >6,1 или 6,0 года среди получавших один, два или три ПССП. К моменту, когда интенсификация наконец произошла, средний $\mathrm{HbA}_{1 c}$ находился между 8,7 и 9,7\%. Эти наблюдения ясно показывают, что существует большая задержка 
в интенсификации терапии у пациентов с СД2, несмотря на неоптимальный контроль гликемии, и что значительная доля пациентов находится в декомпенсации в течение нескольких лет до момента, когда возникает вопрос об интенсификации лечения. В результате такой отложенной коррекции сахароснижающей терапии пациенты неоправданно подвергаются гипергликемии и повышенному риску развития осложнений СД.

В недавнем исследовании Laiteerapong и соавт. [4] определили влияние отсроченного контроля гликемии в когортном исследовании на 34737 людях с впервые выявленным СД2. Авторы изучили взаимосвязи между $\mathrm{HbA}_{1 с}<6,5 \%$ (<48 ммоль/моль), от 6,5\% до $<7,0 \%$ (от 48 до $<53$ ммоль/моль), от 7,0\% до $<8,0 \%$ (от 53 до <64 ммоль/моль), от 8,0\% до $<9,0 \%$ (от 64 до <75 ммоль/моль) или >9,0\% (>75 ммоль/моль) с различными периодами раннего воздействия (0-1, 0-2, 0-3, 0-4, 0-5, 0-6 и 0-7 лет), встречаемостью микрои макрососудистых осложнений и смертностью у данных пациентов при средней длительности наблюдения 13 лет. В сравнении с $\mathrm{HbA}_{1 c}<6,5 \%$ (<48 ммоль/моль) при экспозиции длительность 0-1 год $\mathrm{HbA}_{1 c}>6,5 \%$ (>48 ммоль/моль) были ассоциированы с более частыми микрососудистыми и макрососудистыми событиями, a $\mathrm{HbA}_{1 c}>7,0 \%$ (>53 ммоль/моль) ассоциировались с повышенной смертностью (рис. 2). Данные результаты подкрепляют идею о том, что немедленное лечение, нацеленное на тщательный и длительный контроль гликемии сразу после постановки диагноза, необходимо для предотвращения долгосрочного риска развития осложнений диабета и повышенной смертности.

\section{САХАРНЫЙ ДИАБЕТ 2 ТИПА - МНОГОФАКТОРНОЕ ЗАБОЛЕВАНИЕ}

Прошлые схемы лечения не помогли в борьбе с клинической «инертностью», используя ступенчатый подход, т.е. если добавлять препарат при неэффективности предыдущего(их), это может привести к значительному запаздыванию [3]. Кроме того, ступенчатый подход не подразумевает сложного патогенеза СД2. Нам понадобилось долгое время для того, чтобы принять центральное место нарушения секреции инсулина и инсулинорезистентности [5] в развитии заболевания. Еще больше времени потребовалось, чтобы понять, что другие механизмы, такие как гиперактивность альфа-клеток, недостаточность инсулина/инсулинорезистентность, неадекватная реабсорбция глюкозы в почках и нарушение интегративной деятельности мозга могут участвовать в нарушении гомеостаза глюкозы и способствовать возникновению и прогрессированию гипергликемии [6].

Столь сложный патогенез дает возможность понять, что эффективное лечение может требовать препаратов, действующих на несколько патогенетических механизмов. Современные рекомендации советуют комбинированную терапию с момента постановки диагноза только в случае выраженной декомпенсации углеводного обмена (т.е. $\mathrm{HbA}_{1 c}>9 \%$ ) $[7,8]$. Тем не менее большинство рекомендаций призывают к упреждающему подходу к снижению гликемии при СД2. В изложении позиции ADA/EASD, к примеру, в качестве стартового лечения рекомендуется монотерапия метформином, но предлагает- ся рассмотреть вопрос о назначении второго препарата, если целевой $\mathrm{HbA}_{1 c}$ не достигается за 3 мес лечения [7]. Тем же образом для двух препаратов: при невозможности достичь целевого $\mathrm{HbA}_{1 c}$ в течение 3 мес следует рассмотреть назначение третьего. Очевидно, что большая доля пациентов с СД2 находились бы на комбинированной терапии намного раньше, если бы эти рекомендации неукоснительно соблюдались. Хотя раннее начало комбинированной терапии может с большей вероятностью обеспечить достижение надежной компенсации [9], существует малое количество рекомендаций для клиницистов касательно того, как выбрать оптимальные сочетания препаратов. Это немаловажный повод для размышления, поскольку в настоящее время доступно 9 классов сахароснижающих препаратов и 36 возможных вариантов сочетания для двухкомпонентной и 84 варианта для трехкомпонентной терапии.

Более обоснованный выбор комбинированной терапии предполагает более основательный научный подход и более тщательно выработанные клинические данные. Возможно, в ближайшем будущем более точное фенотипическое определение каждого пациента, а также более сложная обработка клинических и личных данных (т.е. точная медицина) будут помогать нам в принятии таких сложных решений [10]. В настоящее время следующий анализ компонентов может быть достаточным для более осмысленного выбора комбинаций препаратов.

1. Патофизиологическая основа заболевания.

2. Взаимодополняющие механизмы действия.

3. Соотношение эффективность-безопасность.

4. Внегликемические свойства сахароснижающих препаратов.

\section{ПАТОФИЗИОЛОГИЧЕСКАЯ ОСНОВА ЗАБОЛЕВАНИЯ}

По сравнению с предыдущими схемами лечения, в настоящее время мы располагаем препаратами, действующими на более определенные механизмы, обусловливающие развитие диабетической гипергликемии. С годами мы переместились от случайного открытия сахароснижающих свойств препаратов, таких как производные сульфонилмочевины и бигуаниды, к тому времени, когда разработка лекарств для лечения диабета чаще берет начало из более глубокого понимания патофизиологии нарушенного гомеостаза глюкозы. Поэтому применение сахароснижающих препаратов в современном мире не должно просто опираться на их эмпирическую эффективность, но также разумно основываться на коррекции или улучшении определенных механизмов.

Метформин в настоящее время рекомендуется В качестве первой линии терапии СД2 [7, 8]. Препарат главным образом действует путем улучшения чувствительности к инсулину на уровне печени, увеличивая инсулин-индуцированную супрессию продукции глюкозы, при этом мало влияя на чувствительность периферических тканей к инсулину (мышц скелета и жировой ткани) [11]. Рациональный подход к комбинированной терапии закономерно приведет к использованию препарата(ов), улучшающих функцию бета-клеток. Метформин, среди множества свойств, также действует как усилитель глюкагоноподобного пептида-1 (ГПП-1). Как описано Cho 

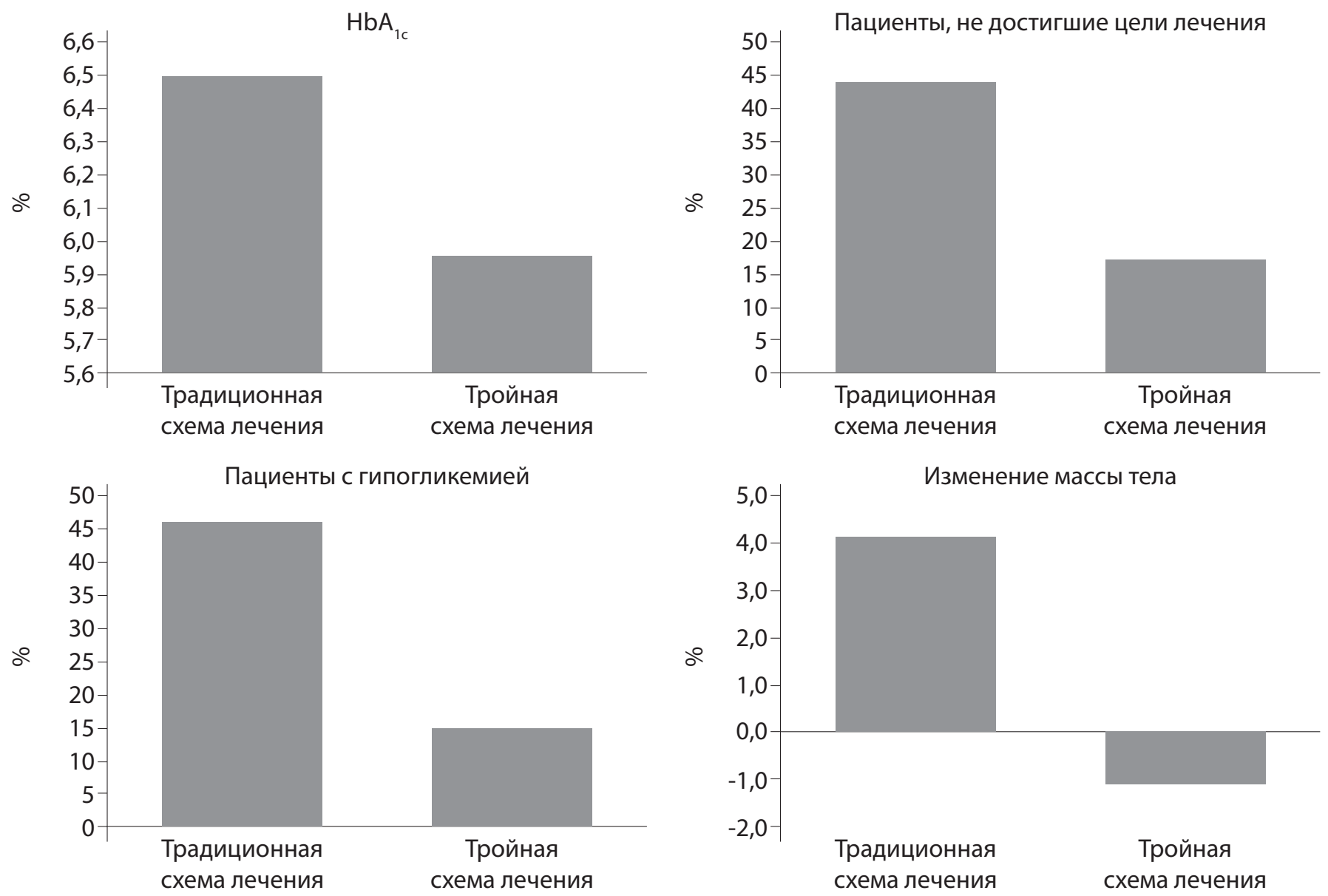

Рис. 3. Результаты сравнения двух групп участников исследования (традиционная схема лечения - начало терапии с повышающихся доз метформина с последующим дополнением препаратов сульфонилмочевины и инсулина гларгина; тройная схема лечения - трехкомпонентная терапия метформин/пиоглитазон/эксенатид с начала лечения) от исходной точки после двух лет лечения по традиционной или тройной схеме лечения. Критерий недостижения цели лечения: $\mathrm{HbA}_{1 c}>6,5 \%$ на двух последовательных визитах с интервалом в 3 мес, несмотря на максимальные дозы сахароснижающих препаратов. Гипогликемия: снижение гликемии <3,3 ммоль/л (60 мг/дл) или появление характерных симптомов. Средний $\mathrm{HbA}_{1 с}$ в начале исследования - 8,6\%, средняя масса тела - 101 кг.

и соавт. [12], метформин может увеличивать экспрессию гена ГПП-1 в L-клетках кишечника и повышать чувствительность бета-клеток к действию ГПП-1. В связи с этим ингибитор дипептидилпептидазы-4 (иДПП-4) выглядит естественным спутником метформина вследствие его способности сохранения эндогенного ГПП-1. Хотя главным образом ГПП-1 продуцируется в дистальной части кишечника, некоторое количество может синтезироваться и местно -высвобождаться альфа-клетками поджелудочной железы [13] в ответ на метаболические изменения [14]. Стоит отметить, что ДПП-4, фермент, отвечающий за разрушение ГПП-1, также экспрессируется альфа-клетками [15]. Поэтому заманчиво предположить, что иДПП-4 могут участвовать в поддержании повышенной внутриостровковой концентрации ГПП-1 и за счет этого способствовать сохранению пула функционирующих бета-клеток. Существование подобной возможности подкрепляется данными нескольких доклинических исследований [16-19], хотя исследования у людей ограничиваются заключениями о том, что применение иДПП-4, с или без метформина, может улучшать функционирование бета-клеток за счет улучшения их чувствительности [20]. Более того, иДПП-4 могут одновременно восстановить глюкозозависимую супрессию глюкагона, восстанавливая более физиологический внутриостровковый гормональный баланс [20]. Действие иДПП-4 также, очевидно, может быть достигнуто и при использовании агонистов рецептора ГПП-1 (аГПП-1) [21]. Данные препараты также положительно действуют на массу тела [21]. Сходства и различия иДПП-4 и аГПП-1 можно использовать для персонализации лечения: ДПП-4 можно рассматривать для поддержания массы тела, аГПП-1 для ее снижения.

По тому же принципу пиоглитазон можно рассматривать как альтернативу метформину, в случае если необходима большая чувствительность периферических тканей к инсулину [11]. Важно, что тиазолидиндионы также могут оказывать защитное действие в отношении бета-клеток [22]. Мы ранее показали, что росиглитазон способен защищать островки поджелудочной железы от липотоксичности [23]. С клинической точки зрения, начальная комбинация пиоглитазона и иДПП-4 показала себя эффективной и хорошо переносимой [24].

Резюмируя, сахароснижающие препараты могут использоваться и комбинироваться на основании их фармакологических мишеней. Недавно Abdul-Ghani и соавт. расширили и опробовали данный подход [25]. В исследование приглашали ранее не получавших лечения пациентов с недавно диагностированным СД2. Участников исследования рандомизировали на две группы - на трехкомпонентную терапию метформин/пиоглитазон/эксенатид или классический ступенчатый подход, начиная с повышающихся доз метформина, затем с добавлением производных сульфонилмочевины и инсулина гларгин. Целью лечения было поддержание $\mathrm{HbA}_{1 c}<6,5 \%$ в течение 2 лет (рис. 3). У пациентов с СД2, которые начинали с трехкомпонентной 
терапии, отмечалось более выраженное снижение $\mathrm{HbA}_{1 c^{\prime}}$ по сравнению с пациентами на традиционной схеме $(5,95$ vs. $6,50 \%$; $p<0,001$ ) с преимуществом в виде в 7,5 раза более низкой частотой гипогликемий и средним снижением массы тела на 1,2 кг, тогда как в группе традиционной схемы отмечался набор массы тела в среднем на 4,1 кг $(\mathrm{p}<0,01)$. Результаты данного поискового исследования показывают, что комбинация препаратов, нацеленная на улучшение функции бета-клеток (эксенатид), увеличение инсулинзависимой утилизации глюкозы (пиоглитазон) и подавление продукции глюкозы печенью (метформин) более эффективна, чем классический ступенчатый подход.

\section{ВЗАИМОДОПОЛНЯЮЩИЕ МЕХАНИЗМЫ ДЕЙСТВИЯ}

Описанное выше уже является примером взаимодополняющих механизмов действия. Используя этот подход, можно ожидать большую эффективность и, возможно, большую длительность компенсации. Однако сочетание механизмов действия также может быть причиной возникновения или усугубления нежелательных эффектов сахароснижающих препаратов. Лечение ингибиторами натрий-глюкозного котранспортера-2 (SGLT2i) ассоциировано с повышением концентрации глюкагона плазмы и парадоксальным повышением эндогенной продукции глюкозы $[26,27]$. Последнее может в некоторой степени оттенять сахароснижающую эффективность данных препаратов, поэтому если предотвратить повышение эндогенной продукции глюкозы, можно ожидать улучшения эффективности препарата. Метформин, как уже говорилось, главным образом действует на уровне печени, и в доклинических исследованиях показано, что он может нейтрализовать персистенцию продукции глюкозы печенью, запускаемую SGLT2i [28]. В соответствии с этим биологическим экспериментом клинические исследования показали превосходство комбинации метформина и SGLT2i в контроле гликемии [29].

Препараты инкретинового ряда могут снижать постпрандиальную секрецию глюкагона. Как заявляется, этот эффект может обусловливать до 50\% супрессии продукции глюкозы печенью при назначении эксенатида [30]. Hansen и соавт. показали, что применение иДПП-4 (саксаглиптина) вместе с метформином и SGLT2i (дапаглифлозином) предотвращало постпрандиальное повышение уровня глюкагона (что наблюдалось при использовании метформина и дапаглифлозина), также улучшая постпрандиальную чувствительность к глюкозе [31]. Согласно этим результатам, в исследованиях, где сравнивались комбинации иДДП-4 [32, 33] или аГПП-1 [34, 35] с SGLT2i и монотерапия, первые показали несколько лучшие значения $\mathrm{HbA}_{1 c}$ и значительно большую долю пациентов, достигших целевого уровня - <7,0\%.

Таким образом, препараты с взаимодополняющими механизмами действия могут использоваться для потенцирования сахароснижающей эффективности или для предотвращения метаболических изменений, ограничивающих фармакологический потенциал препарата.

\section{СООТНОШЕНИЕ ЭФФЕКТИВНОСТЬ-БЕЗОПАСНОСТЬ}

По данным мета-анализа, проведенного Phung и соавт. [9], начало лечения с комбинации препаратов у пациентов с СД2, ранее не получавших лечения, ассоциировано с лучшим, по сравнению с монотерапией метформином, контролем гликемии, поэтапным снижением $\mathrm{HbA}_{1 с}$ на 0,43\% и на 40\% более высоким шансом достигнуть целевого $\mathrm{HbA}_{1 c}$ (7,0\%). Дальнейший анализ также оценивал эффективность отдельных препаратов, добавленных к метформину. Palmer и соавт. провели тщательный сравнительный анализ эффективности препаратов, добавленных к метформину [36]. Среди взрослых с СД2 не было значительных различий между любыми из 9 доступных классов сахароснижающих препаратов. Хотя эффективность обычно оценивается по снижению $\mathrm{HbA}_{1 c^{\prime}}$ также важно учитывать долговечность этого действия. С этой точки зрения, тиазолидиндионы неоднократно описывались как препараты с более долговечным эффектом, чем метформин и особенно производные сульфонилмочевины [37], хотя для такого лечения необходим внимательный подбор пациентов из-за вероятности задержки жидкости, риска сердечной недостаточности и патологических переломов костей. иДПП4 оценивались в качестве дополнения к метформину при лечении в течение 2 лет и в общем показали сходную с производными сульфонилмочевины эффективность [38], и в одном 104-недельном исследовании отмечено умеренное, но достоверно большее улучшение показателей $\mathrm{HbA}_{1 с}$ к окончанию исследования [39]. В более позднем исследовании длительностью до 4 лет изучалась долговечность дапаглифлозина в качестве дополнительной терапии к метформину в сравнении с глипизидом [40]. В группе дапаглифлозина отмечался достоверно меньший коэффициент неудачи по сравнению с группой глипизида (0,19 [95\% ДИ 0,12-0,25] vs. 0,61 [95\% ДИ 0,49-0,72]), а также в 10 раз меньшая частота гипогликемий. Применение дапаглифлозина также было связано со стойким снижением массы тела и артериального давления [40]. Palmer и соавт. в проведенном мета-анализе также оценивали относительный риск гипогликемии и увеличения массы тела для отдельных препаратов в комбинации с метформином [36]. SGLT2i имели самые низкие шансы гипогликемий, а аГПП-1, при добавлении к метформину и производным сульфонилмочевины, ассоциировались с наименьшим риском гипогликемий. Производные сульфонилмочевины и пиоглитазон были связаны с повышенным риском набора массы тела, в то время как SGLT2i и аГПП-1 были ассоциированы с меньшим набором массы тела, если не с ее снижением. Данные наблюдения важны, поскольку при выборе сахароснижающего препарата для комбинации, помимо эффективности, необходимо оценивать возможные взаимодействия и безопасность. К примеру, значимое достоверное увеличение риска гипогликемий было обнаружено при комбинированной терапии в сравнении с монотерапией метформином [отношение рисков (OP) 1,56 (1,08-2,26)], но этот эффект был незначимым при исключении из анализа исследований, где метформин сочетался с производными сульфонилмочевины или глинидами [OP 1,20 (0,91-1,56)] [9]. Другая комбинация может быть более нейтральной [41] и помочь сгладить побочные эффекты, как в случае добавления SGLT2і к пиоглитазону [42]. В этом случае отмечались повышенная эффективность и сглаживание типичного для пиоглитазона набора веса. Более того, эффект осмотического ди- 
Таблица 1. Потенциальные положительные эффекты раннего начала комбинированного лечения сахарного диабета 2 типа и нерешенные в настоящее время вопросы

\section{Польза}

Рациональный подход к лечению

Воздействует на несколько звеньев патогенеза

Преимущество взаимодополняющего действия препаратов

Возможность установления баланса между эффективностью и побочными эффектами, что открывает возможности для индивидуализированного лечения

Может способствовать более стойкой эффективности и дополнительному положительному влиянию в виде снижения долгосрочного риска осложнений
Boпрос

Насколько длительное

поддержание компенсации?

Может ли улучшить приверженность к лечению?

Может ли снизить клиническую «инертность» врача?

Позволит ли сохранить функцию бета-клеток?

Будет ли стоимость лечения подходящей? уреза SGLT2i также может снижать задержку жидкости, и к тому же, оба препарата не связаны с риском гипогликемий [43]. В ходе недавнего post-hoc-анализа, в котором оценивалась безопасность трехкомпонентной пероральной сахароснижающей терапии метформин/ саксаглиптин/дапаглифлозин vs. двухкомпонентной терапии метформином и дапаглифлозином или саксаглиптином, было обнаружено, что частота встречаемости нежелательных и серьезных нежелательных явлений была сходной в обеих группах [44]. Примечательно, что инфекции мочевыводящих путей отмечались чаще при последовательном, чем при одномоментном назначении препаратов, а генитальные инфекции отмечены только при последовательном добавлении дапаглифлозина к комбинации саксаглиптина и метформина [44].

Тщательная оценка отношения риск-польза при раннем назначении комбинированной терапии - ключ к достижению хорошей комплаентности. В этой связи доступность комбинированных препаратов с фиксированными дозами позволит снизить количество принимаемых таблеток и таким образом улучшить приверженность пациента к лечению [45].

Множество возможных комбинаций препаратов может приводить к различным показателям отношения риск-польза. Это может в дальнейшем стать препятствием в лечении СД2, но также и возможностью для более индивидуализированного лечения.

\section{ВНЕГЛИКЕМИЧЕСКИЕ СВОЙСТВА}

Хотя контроль гликемии остается ключевым фактором снижения риска осложнений диабета, некоторые сахароснижающие препараты могут иметь дополнительные свойства, позволяющие достичь большей защиты. Классический пример - пиоглитазон. После того как этот инсулинсенсибилизирующий препарат был представлен как сахароснижающий, скоро стало очевидно, что он также обладает другими свойствами, связанными с антиатерогенным действием [46]. В исследовании ProActive [47] пиоглитазон исследовался в аспекте кардиопротективного действия. Хотя главная конечная точка (нефатальный инфаркт миокарда, нефатальный инсульт, сердечно-сосудистая смертность и реваскуляризация) не достигла статистической достоверности, вторичная конечная точка (те же самые параметры, что и в первичной, за исключением реваскуляризации) достигла стати- стической значимости (ОР 0,84; ДИ 0,72-0,98; $\mathrm{p}=0,027$ ). Исследование IRIS [48] подтвердило это кардиопротективное свойство и подкрепило возможность внегликемического действия, так как исследование проводилось среди пациентов с инсулинорезистентностью без СД.

В последние годы отмечалась корреляция кардиопротективного эффекта с применением SGLT2i [49, 50] и аГПП-1 [51-53]. Стоит отметить, что механизмы этого действия могут различаться у трех классов препаратов: главным образом антиатерогенный для пиоглитазона, гемодинамический и метаболический для SGLT2i и потенциально прямое действие на кардиомиоциты и сосуды для аГПП-1 [54]. Если это действительно так, эти наблюдения позволят начать новые исследования возможного взаимодействия при применении этих препаратов в комбинации: не только в сахароснижающем аспекте, но и потенцировании кардиопротективных свойств.

Признание и доказательство того, что некоторые сахароснижающие препараты могут способствовать предотвращению микрососудистых осложнений, еще один важный аспект в вопросе раннего начала комбинированной терапии. К примеру, утверждается, что иДПП-4 имеют несколько эффектов, которые могут способствовать сохранению микроциркуляции [55]. Сходным образом показаны нефропротективные свойства аГПП-1 [56], даже более заметные у SGLT2i [57]. В настоящее время проводятся определенные исследования для изучения данного потенциала этих препаратов. Если исследования подтвердят эти свойства, нетрудно будет поставить вопрос о введении этих препаратов в схемы ранней комбинированной терапии для лучшего и более постоянного контроля гликемии и в то же время иметь возможность защиты от сосудистых осложнений.

\section{ЗАКЛЮЧЕНИЕ}

Современное лечение СД2 требует изменения парадигмы в виде соответствующей интенсификации терапии от самой постановки диагноза. Определение многокомпонентности патогенеза СД2 приводит к пониманию необходимости воздействия на заболевание с разных сторон, т.е. одновременного действия на несколько механизмов развития гипергликемии. Самым важным аспектом в снижении риска осложнений диабета является быстрое достижение компенсации углеводного обмена и ее поддержание. Поэтому комбинированная 
терапия должна применяться если не при постановке диагноза, то как минимум активным и упреждающим образом, чтобы избежать экспозиции пациента даже при легкой хронической гипергликемии и обеспечить раннее и стойкое положительное влияние (табл. 1). Хотя такой подход выглядит разумным и целесообразным, остаются вопросы, на которые необходимо ответить (см. табл. 1). Прежде всего, нам необходимы более весомые доказательства для подтверждения целесообразности подхода и рекомендации по возможным комбинациям препаратов для конкретных пациентов. Кроме того, нам понадобится определить, может ли начало лечения с комбинации препаратов изменять, улучшать и поддерживать крайне важные патофизиологические механизмы, такие как функции бета-клеток, ожидая, что это позволит достигать более стойкого контроля гликемии; нам понадобится оценить, насколько комбинированная терапия может повлиять на приверженность пациентов лечению и клиническую инертность врачей - два главных фактора потери контроля гликемии с течением времени. Наконец, тщательная оценка баланса затрат и эффективности будет необходима для определения жизнеспособности более дорогой стартовой терапии.

В заключение предстоит проделать много работы, но некоторые исследования уже ведутся. Некоторые из поднятых вопросов будут изучаться в исследованиях GRADE (Glycemia Reduction Approaches in Diabetes: A Comparative Effectiveness Study) [58] и VERIFY (Vildagliptin Efficacy in with metfoRmln For earlY treatment of type 2 diabetes mellitus) [59]. В исследовании GRADE будут сравнивать производные сульфонилмочевины, иДПП-4, аГПП-1 и базальный инсулин в качестве дополнительной терапии к метформину у пациентов с недавно (<5 лет) диагностированным СД2, чтобы убедиться в поддержании метаболической компенсации, изучить нежелательные явления, влияние на сердечно-сосудистые факторы риска, переносимость и соотношение стоимость-эффективность. Однако SGLT2i не были включены в исследование, что препятствует изучению потен- циала этого класса в ранней комбинированной терапии. Исследование VERIFY позволит изучить долгосрочную клиническую пользу ранней комбинированной терапии метформином и вилдаглиптином (иДПП-4) в сравнении со ступенчатым применением тех же препаратов у пациентов с недавно выявленным СД2 и небольшим повышением $\mathrm{HbA}_{1 c^{\prime}}$ для сравнения долгосрочности гликемического контроля, функции бета-клеток и чувствительности к инсулину, время до подключения к терапии инсулина и влияние на диабетические осложнения в ходе пятилетнего наблюдения.

Пока мы ждем результатов этих и будущих исследований, нам необходимо осознать, что СД2 - тяжелое состояние в любой стадии заболевания, включая раннюю, даже в присутствии небольшого повышения уровня глюкозы. Поэтому все потенциальные способы уменьшить тяжесть заболевания должны тщательно рассматриваться.

\section{ДОПОЛНИТЕЛЬНАЯ ИНФОРМАЦИЯ}

Источники финансирования. Публикация данной статьи на русском и английском языках поддержана Novartis Pharma AG. Спонсор не принимал участие в проведении поисково-аналитической работы, анализе и интерпретации данных и подготовке статьи.

Конфликт интересов. Stefano Del Prato получал поддержку исследования от AstraZeneca, MSD, Novartis and Boehringer Ingelheim, и платежи от AstraZeneca, Boehringer Ingelheim, Eli Lilly, GSK, MSD, Mundipharma, Novartis, Novo Nordisk, Sanofi, Servier и Takeda за консультации.

Francesco Indovina и Pierpaolo Falcetta декларируют отсутствие явных и потенциальных конфликтов интересов.

Вклад авторов. Francesco Indovina, Pierpaolo Falcetta собирали данные, Francesco Indovina, Pierpaolo Falcetta и Stefano Del Prato провели анализ и написали статью. Все авторы внесли существенный вклад в проведение исследования и подготовку статьи, прочли и одобрили финальную версию перед публикацией.

Данная статья является резюме лекции Stefano Del Prato, представленной на научной конференции Novartis 27 апреля 2018 года в Санкт-Петербурге.

\section{СПИСОК ЛИТЕРАТУРЫ | REFERENCES}

1. Tahrani AA, Bailey CJ, Del Prato S, Barnett AH. Management of type 2 diabetes: new and future developments in treatment. Lancet. 2011;378(9786):182-197. doi: 10.1016/s0140-6736(11)60207-9

2. Carls G, Huynh J, Tuttle E, et al. Achievement of Glycated Hemoglobin Goals in the US Remains Unchanged Through 2014. Diabetes Ther. 2017;8(4):863-873. doi: 10.1007/s13300-017-0280-5

3. Khunti K, Wolden ML, Thorsted BL, et al. Clinical inertia in people with type 2 diabetes: a retrospective cohort study of more than 80,000 people. Diabetes Care. 2013;36(11):3411-3417. doi: 10.2337/dc13-0331

4. Laiteerapong N, Ham SA, Gao Y, et al. The Legacy Effect in Type 2 Diabetes: Impact of Early Glycemic Control on Future Complications (the Diabetes \& Aging Study). Diabetes Care. 2018. doi: 10.2337/dc17-1144 Kahn SE, Cooper ME, Del Prato S. Pathophysiology and treatment of type 2 diabetes: perspectives on the past, present, and future. Lancet. 2014;383(9922):1068-1083. doi: 10.1016/s0140-6736(13)62154-6

6. Defronzo RA. Banting Lecture. From the triumvirate to the ominous octet: a new paradigm for the treatment of type 2 diabetes mellitus. Diabetes. 2009;58(4):773-795. doi: 10.2337/db09-9028

7. Inzucchi SE, Bergenstal RM, Buse JB, et al. Management of hyperglycaemia in type 2 diabetes, 2015: a patient-centred approach. Update to a position statement of the American Diabetes Association and the European Association for the Study of Diabetes. Diabetologia. 2015;58(3):429-442. doi: 10.1007/s00125-014-3460-0
8. Garber AJ, Abrahamson MJ, Barzilay Jl, et al. Consensus Statement by the American Association of Clinical Endocrinologists and American College of Endocrinology on the Comprehensive Type 2 Diabetes Management Algorithm--2016 Executive Summary. Endocr Pract. 2016;22(1):84-113. doi: 10.4158/EP151126.CS

9. Phung OJ, Sobieraj DM, Engel SS, Rajpathak SN. Early combination therapy for the treatment of type 2 diabetes mellitus: systematic review and meta-analysis. Diabetes Obes Metab. 2014;16(5):410-417. doi: 10.1111/dom.12233

10. Gloyn AL, Drucker DJ. Precision medicine in the management of type 2 diabetes. Lancet Diabetes Endocrinol. 2018. doi: 10.1016/s2213-8587(18)30052-4

11. Natali A, Ferrannini E. Effects of metformin and thiazolidinediones on suppression of hepatic glucose production and stimulation of glucose uptake in type 2 diabetes: a systematic review. Diabetologia. 2006;49(3):434-441. doi: 10.1007/s00125-006-0141-7

12. Cho YM, Kieffer TJ. New aspects of an old drug: metformin as a glucagon-like peptide 1 (GLP-1) enhancer and sensitiser. Diabetologia. 2011;54(2):219-222. doi: 10.1007/s00125-010-1986-3

13. Marchetti P, Lupi R, Bugliani M, et al. A local glucagon-like peptide 1 (GLP-1) system in human pancreatic islets. Diabetologia. 2012;55(12):3262-3272. doi: 10.1007/s00125-012-2716-9 
14. Sancho V, Daniele G, Lucchesi D, et al. Metabolic regulation of GLP-1 and PC1/3 in pancreatic alpha-cell line. PLoS One. 2017;12(11):e0187836. doi: 10.1371/journal.pone.0187836

15. Poulsen MD, Hansen GH, Dabelsteen E, et al. Dipeptidyl peptidase IV is sorted to the secretory granules in pancreatic islet A-cells. J Histochem Cytochem. 1993;41(1):81-88. doi: 10.1177/41.1.8093256

16. Inaba W, Mizukami H, Kamata K, et al. Effects of long-term treatment with the dipeptidyl peptidase-4 inhibitor vildagliptin on islet endocrine cells in non-obese type 2 diabetic Goto-Kakizaki rats. Eur J Pharmacol. 2012;691(1-3):297-306. doi: 10.1016/j.ejphar.2012.07.030

17. Mu J, Petrov A, Eiermann GJ, et al. Inhibition of DPP-4 with sitagliptin improves glycemic control and restores islet cell mass and function in a rodent model of type 2 diabetes. Eur J Pharmacol. 2009;623(1-3):148-154. doi: 10.1016/j.ejphar.2009.09.027

18. Furuta $Y$, Horiguchi $M$, Sugaru $E$, et al. Chronic administration of DSP-7238, a novel, potent, specific and substrate-selective DPP IV inhibitor, improves glycaemic control and beta-cell damage in diabetic mice. Diabetes Obes Metab. 2010;12(5):421-430. doi: 10.1111/j.1463-1326.2009.01180.x

19. Shah P, Ardestani A, Dharmadhikari G, et al. The DPP-4 inhibitor linagliptin restores beta-cell function and survival in human isolated islets through GLP-1 stabilization. J Clin Endocrinol Metab. 2013;98(7):E1163-1172. doi: 10.1210/jc.2013-1029

20. Muscelli E, Casolaro A, Gastaldelli A, et al. Mechanisms for the antihyperglycemic effect of sitagliptin in patients with type 2 diabetes. J Clin Endocrinol Metab. 2012;97(8):2818-2826. doi: 10.1210/jc.2012-1205

21. Nauck MA, Meier JJ. The incretin effect in healthy individuals and those with type 2 diabetes: physiology, pathophysiology, and response to therapeutic interventions. Lancet Diabetes Endocrinol. 2016:4(6):525-536. doi: 10.1016/s2213-8587(15)00482-9

22. Gastaldelli A, Ferrannini E, Miyazaki Y, et al. Thiazolidinediones improve beta-cell function in type 2 diabetic patients. Am J Physiol Endocrinol Metab. 2007;292(3):E871-883. doi: 10.1152/ajpendo.00551.2006

23. Lupi R, Del Guerra S, Marselli L, et al. Rosiglitazone prevents the impairment of human islet function induced by fatty acids: evidence for a role of PPARgamma2 in the modulation of insulin secretion. Am J Physiol Endocrinol Metab. 2004;286(4):E560-567. doi: 10.1152/ajpendo.00561.2002

24. Rosenstock J, Kim SW, Baron MA, et al. Efficacy and tolerability of initial combination therapy with vildagliptin and pioglitazone compared with component monotherapy in patients with type 2 diabetes. Diabetes Obes Metab. 2007;9(2):175-185. doi: 10.1111/j.1463-1326.2006.00698.x

25. Abdul-Ghani MA, Puckett C, Triplitt C, et al. Initial combination therapy with metformin, pioglitazone and exenatide is more effective than sequential add-on therapy in subjects with new-onset diabetes. Results from the Efficacy and Durability of Initial Combination Therapy for Type 2 Diabetes (EDICT): a randomized trial. Diabetes Obes Metab. 2015;17(3):268-275. doi: 10.1111/dom.12417

26. Merovci A, Solis-Herrera C, Daniele G, et al. Dapagliflozin improves muscle insulin sensitivity but enhances endogenous glucose production. J Clin Invest. 2014;124(2):509-514. doi: 10.1172/JCI70704

27. Ferrannini E, Muscelli E, Frascerra S, et al. Metabolic response to sodium-glucose cotransporter 2 inhibition in type 2 diabetic patients. J Clin Invest. 2014;124(2):499-508. doi: 10.1172/JCI72227

28. Neschen S, Scheerer M, Seelig A, et al. Metformin supports the antidiabetic effect of a sodium glucose cotransporter 2 inhibitor by suppressing endogenous glucose production in diabetic mice. Diabetes. 2015;64(1):284-290. doi: 10.2337/db14-0393

29. Rosenstock J, Chuck L, Gonzalez-Ortiz M, et al. Initial Combination Therapy With Canagliflozin Plus Metformin Versus Each Component as Monotherapy for Drug-Naive Type 2 Diabetes. Diabetes Care. 2016;39(3):353-362. doi: 10.2337/dc15-1736

30. Cervera A, Wajcberg E, Sriwijitkamol A, et al. Mechanism of action of exenatide to reduce postprandial hyperglycemia in type 2 diabetes. Am J Physiol Endocrinol Metab. 2008;294(5):E846-852. doi: 10.1152/ajpendo.00030.2008

31. Hansen L, lqbal N, Ekholm E, et al. Postprandial dynamics of plasma glucose, insulin, and glucagon in patients with type 2 diabetes treated with saxagliptin plus dapagliflozin add-on to metformin therapy. Endocr Pract. 2014;20(11):1187-1197. doi: 10.4158/EP14489.OR

32. Lewin A, DeFronzo RA, Patel S, et al. Initial combination of empagliflozin and linagliptin in subjects with type 2 diabetes. Diabetes Care. 2015;38(3):394-402. doi: 10.2337/dc14-2365
33. Rosenstock J, Hansen L, Zee $\mathrm{P}$, et al. Dual add-on therapy in type 2 diabetes poorly controlled with metformin monotherapy: a randomized double-blind trial of saxagliptin plus dapagliflozin addition versus single addition of saxagliptin or dapagliflozin to metformin. Diabetes Care. 2015;38(3):376-383. doi: 10.2337/dc14-1142

34. Frías JP, Guja C, Hardy E, et al. Exenatide once weekly plus dapagliflozin once daily versus exenatide or dapagliflozin alone in patients with type 2 diabetes inadequately controlled with metformin monotherapy (DURATION-8): a 28 week, multicentre, double-blind, phase 3, randomised controlled trial. Lancet Diabetes Endocrinol. 2016;4(12):1004-1016. doi: 10.1016/s2213-8587(16)30267-4

35. Ludvik B, Frías JP, Tinahones FJ, et al. Dulaglutide as add-on therapy to SGLT2 inhibitors in patients with inadequately controlled type 2 diabetes (AWARD-10): a 24-week, randomised, double-blind, placebo-controlled trial. Lancet Diabetes Endocrinol. 2018;6(5):370-381. doi: 10.1016/s2213-8587(18)30023-8

36. Palmer SC, Mavridis D, Nicolucci A, et al. Comparison of Clinical Outcomes and Adverse Events Associated With Glucose-Lowering Drugs in Patients With Type 2 Diabetes: A Meta-analysis. JAMA. 2016;316(3):313-324. doi: 10.1001/jama.2016.9400

37. Kahn SE, Haffner SM, Heise MA, et al. Glycemic durability of rosiglitazone, metformin, or glyburide monotherapy. N Engl J Med. 2006;355(23):2427-2443. doi: 10.1056/NEJMoa066224

38. Mishriky BM, Cummings DM, Tanenberg RJ. The efficacy and safety of DPP4 inhibitors compared to sulfonylureas as add-on therapy to metformin in patients with Type 2 diabetes: A systematic review and meta-analysis. Diabetes Res Clin Pract. 2015;109(2):378-388. doi: 10.1016/j.diabres.2015.05.025

39. Del Prato S, Camisasca R, Wilson C, Fleck P. Durability of the efficacy and safety of alogliptin compared with glipizide in type 2 diabetes mellitus: a 2-year study. Diabetes Obes Metab. 2014;16(12):1239-1246. doi: 10.1111/dom.12377

40. Del Prato S, Nauck M, Duran-Garcia S, et al. Long-term glycaemic response and tolerability of dapagliflozin versus a sulphonylurea as add-on therapy to metformin in patients with type 2 diabetes: 4-year data. Diabetes Obes Metab. 2015;17(6):581-590. doi: 10.1111/dom.12459

41. Del Prato S, Chilton R. Practical strategies for improving outcomes in T2DM: The potential role of pioglitazone and DPP4 inhibitors. Diabetes Obes Metab. 2018;20(4):786-799. doi: 10.1111/dom.13169

42. Kovacs CS, Seshiah V, Swallow R, et al. Empagliflozin improves glycaemic and weight control as add-on therapy to pioglitazone or pioglitazone plus metformin in patients with type 2 diabetes: a 24-week, randomized, placebo-controlled trial. Diabetes Obes Metab. 2014;16(2):147-158. doi: 10.1111/dom.12188

43. DeFronzo RA, Chilton R, Norton L, et al. Revitalization of pioglitazone: the optimum agent to be combined with a sodium-glucose co-transporter-2 inhibitor. Diabetes Obes Metab. 2016;18(5):454-462. doi: $10.1111 /$ dom. 12652

44. Del Prato S, Rosenstock J, Garcia-Sanchez R, et al. Safety and tolerability of dapagliflozin, saxagliptin and metformin in combination: Posthoc analysis of concomitant add-on versus sequential add-on to metformin and of triple versus dual therapy with metformin. Diabetes Obes Metab. 2018;20(6):1542-1546. doi: 10.1111/dom.13258

45. Hutchins $V$, Zhang B, Fleurence RL, et al. A systematic review of adherence, treatment satisfaction and costs, in fixed-dose combination regimens in type 2 diabetes. Curr Med Res Opin. 2011;27(6):1157-1168. doi: $10.1185 / 03007995.2011 .570745$

46. Cariou B, Charbonnel B, Staels B. Thiazolidinediones and PPARgamma agonists: time for a reassessment. Trends Endocrinol Metab. 2012;23(5):205-215. doi: 10.1016/j.tem.2012.03.001

47. Dormandy JA, Charbonnel B, Eckland DJA, et al. Secondary prevention of macrovascular events in patients with type 2 diabetes in the PROactive Study (PROspective pioglitAzone Clinical Trial In macroVascular Events): a randomised controlled trial. Lancet. 2005;366(9493):1279-1289. doi: 10.1016/s0140-6736(05)67528-9

48. Kernan WN, Viscoli CM, Furie KL, et al. Pioglitazone after Ischemic Stroke or Transient Ischemic Attack. N Engl J Med. 2016;374(14):1321-1331. doi: 10.1056/NEJMoa1506930

49. Zinman B, Wanner C, Lachin JM, et al. Empagliflozin, Cardiovascular Outcomes, and Mortality in Type 2 Diabetes. N Engl J Med. 2015;373(22):2117-2128. doi: 10.1056/NEJMoa1504720

50. Neal B, Perkovic V, Mahaffey KW, et al. Canagliflozin and Cardiovascular and Renal Events in Type 2 Diabetes. N Engl J Med. 2017:377(7):644-657. doi: 10.1056/NEJMoa1611925 
51. Marso SP, Daniels GH, Brown-Frandsen K, et al. Liraglutide and Cardiovascular Outcomes in Type 2 Diabetes. NEngl J Med. 2016;375(4):311-322. doi: 10.1056/NEJMoa1603827

52. Marso SP, Bain SC, Consoli A, et al. Semaglutide and Cardiovascular Outcomes in Patients with Type 2 Diabetes. N Engl J Med. 2016;375(19):1834-1844. doi: 10.1056/NEJMoa1607141

53. Holman RR, Bethel MA, Mentz RJ, et al. Effects of Once-Weekly Exenatide on Cardiovascular Outcomes in Type 2 Diabetes. N Engl J Med. 2017:377(13):1228-1239. doi: 10.1056/NEJMoa1612917

54. Abdul-Ghani M, DeFronzo RA, Del Prato S, et al. Cardiovascular Disease and Type 2 Diabetes: Has the Dawn of a New Era Arrived? Diabetes Care. 2017;40(7):813-820. doi: 10.2337/dc16-2736

55. Avogaro A, Fadini GP. The effects of dipeptidyl peptidase-4 inhibition on microvascular diabetes complications. Diabetes Care. 2014;37(10):2884-2894. doi: 10.2337/dc14-0865
56. Mann JFE, Orsted DD, Brown-Frandsen K, et al. Liraglutide and Renal Outcomes in Type 2 Diabetes. N Engl J Med. 2017;377(9):839-848. doi: 10.1056/NEJMoa1616011

57. Wanner C, Inzucchi SE, Lachin JM, et al. Empagliflozin and Progression of Kidney Disease in Type 2 Diabetes. N Engl J Med. 2016;375(4):323-334. doi: 10.1056/NEJMoa1515920

58. Nathan DM, Buse JB, Kahn SE, et al. Rationale and design of the glycemia reduction approaches in diabetes: a comparative effectiveness study (GRADE). Diabetes Care. 2013;36(8):2254-2261. doi: $10.2337 /$ dc13-0356

59. Del Prato S, Foley JE, Kothny W, et al. Study to determine the durability of glycaemic control with early treatment with a vildagliptin-metformin combination regimen vs. standard-of-care metformin monotherapy-the VERIFY trial: a randomized double-blind trial. Diabet Med. 2014;31(10):1178-1184. doi: 10.1111/dme.12508

\section{ИНФОРМАЦИЯ ОБ АВТОРАХ [AUTHORS INFO]}

*Stefano Del Prato, MD, PhD; address: Nuovo Ospedale Santa Chiara, Via Paradisa, 2, 56124 Pisa, Italy; ORCID: https://orcid.org/0000-0002-5388-0270; e-mail: stefano.delprato@med.unipi.it

Francesco Indovina, MD; ORCID: https://orcid.org/0000-0003-1274-1976; e-mail: indofrancesco@gmail.com Pierpaolo Falcetta, MD; e-mail: falcetta.pierpaolo@gmail.com

\section{ЦИТИРОВАТЬ:}

del Prato S, Indovina F, Falcetta P. Сахарный диабет 2 типа. Комбинированная терапия на старте заболевания // Сахарный диабет. — 2018. — T. 21. — №5. — C. 386-394. doi: 10.14341/DM9867

\section{TO CITE THIS ARTICLE:}

del Prato S, Indovina F, Falcetta P. Type 2 Diabetes Mellitus. From the start - combination therapy. Diabetes Mellitus. 2018;21(5):386-394. doi: 10.14341/DM9867 